\title{
Colorectal mixed adenoneuroendocrine carcinomas and neuroendocrine carcinomas are genetically closely related to colorectal adenocarcinomas
}

Moritz Jesinghaus ${ }^{1,9}$, Björn Konukiewitz ${ }^{1,9}$, Gisela Keller ${ }^{1}$, Matthias Kloor ${ }^{2}$, Katja Steiger ${ }^{1}$, Magdalena Reiche ${ }^{1}$, Roland Penzel ${ }^{3}$, Volker Endris ${ }^{3}$, Ruza Arsenic ${ }^{4}$, Gratiana Hermann ${ }^{5}$, Albrecht Stenzinger ${ }^{3,6,7}$, Wilko Weichert ${ }^{1,6,8}$, Nicole Pfarr ${ }^{1,10}$ and Günter Klöppel ${ }^{1,10}$

${ }^{1}$ Institute of Pathology, Technical University of Munich, Munich, Germany; ${ }^{2}$ Applied Tumor Biology, Institute of Pathology, University Hospital Heidelberg, Heidelberg, Germany; ${ }^{3}$ Institute of Pathology, Charité University Medicine, Berlin, Germany; ${ }^{4}$ Institute of Pathology, University Hospital Heidelberg, Heidelberg, Germany; ${ }^{5}$ Department of Pathology, Assaf Harofeh Medical Center, Tel-Aviv University Sackler School of Medicine, Zerifin, Israel; ${ }^{6}$ National Center for Tumor Diseases (NCT), Heidelberg, Germany; ${ }^{7}$ National Center for Tumor Diseases - Heidelberg School of Oncology (NCT-HSO), Heidelberg, Germany and ${ }^{8}$ German Cancer Consortium (DKTK), Heidelberg, Germany

Colorectal mixed adenoneuroendocrine carcinomas are rare and clinically aggressive neoplasms with considerable morphological heterogeneity. Data on their genomic characteristics and molecular associations to either conventional colorectal adenocarcinomas or poorly differentiated neuroendocrine neoplasms is still scarce, hampering optimized patient treatment and care. Tissue from 19 colorectal mixed adenoneuroendocrine carcinomas and eight colorectal poorly differentiated neuroendocrine neoplasms (neuroendocrine carcinomas) was microdissected and subjected to next-generation sequencing using a colorectal adenocarcinoma-specific panel comprising 196 amplicons covering 32 genes linked to colorectal adenocarcinoma, and poorly differentiated neuroendocrine neoplasm tumorigenesis. Mixed adenoneuroendocrine carcinomas were also examined for microsatellite instability and $M L H-1$ promoter methylation status. In three mixed adenoneuroendocrine carcinomas, exocrine and endocrine components were analyzed separately. Genetic testing of colorectal mixed adenoneuroendocrine carcinomas identified 43 somatic mutations clustering in $13 / 32$ genes. Sixteen (84\%) tumors harbored at least one somatic mutation, two tumors $(11 \%)$ displayed high microsatellite instability. Compared with colorectal adenocarcinomas, mixed adenoneuroendocrine carcinomas were more frequently $B R A F(37 \% ; P=0.006)$, and less frequently $\operatorname{KRAS}(21 \% ; P=0.043)$ and $A P C(16 \% ; P=0.001)$ mutated. Point mutations in neuroendocrine neoplasmrelated genes like $R B 1$ or $R E T$ were not detected, but one tumor harbored a heterozygous $R B 1$ deletion. Separately analyzed adenocarcinoma and neuroendocrine carcinoma components revealed a shared mutational trunk of driver genes involved in colorectal adenocarcinoma carcinogenesis. Colorectal neuroendocrine carcinomas were similar in their mutation profile to colorectal adenocarcinomas, but compared with mixed adenoneuroendocrine carcinomas, had a higher rate of $A P C$ mutations $(P=0.027)$. Our data indicate that colorectal mixed adenoneuroendocrine carcinomas and neuroendocrine carcinomas are genetically closely related to colorectal adenocarcinomas, suggesting that the cells giving rise to these tumors primarily have an intestinal coinage. The identification of $B R A F$ mutations and the frequently present $K R A S$ wild-type status principally render some mixed adenoneuroendocrine carcinomas eligible to targeted treatment strategies used for colorectal adenocarcinomas. Modern Pathology (2017) 30, 610-619; doi:10.1038/modpathol.2016.220; published online 6 January 2017

Correspondence: Dr M Jesinghaus, MD, Institute of Pathology, Technical University of Munich, Trogerstrasse 18, 81675 Munich, Germany,

E-mail: moritz.jesinghaus@tum.de

${ }^{9}$ Shared first authorship.

${ }^{10}$ Shared last authorship.

Received 18 July 2016; revised 7 November 2016; accepted 8 November 2016; published online 6 January 2017
Colorectal mixed adenoneuroendocrine carcinomas are rare and heterogeneous neoplasms. They are composed of an exocrine and a neuroendocrine component, per definition each representing at least $30 \%$ of the entire tumor cell population. ${ }^{1-4}$ The cells of these components ${ }^{5}$ either form monodifferentiated complexes that combine with each other in a mosaic 
pattern, or are intimately intermingled and may even show amphicrine features. ${ }^{3,6}$ These characteristics make mixed adenoneuroendocrine carcinomas diagnostically challenging, as only appropriate immunohistochemical screening for neuroendocrine differentiation may reveal the tumor's dual nature. ${ }^{3}$

Clinically, colorectal mixed adenoneuroendocrine carcinomas are usually aggressive neoplasms, ${ }^{7-9}$ with a clinical need for intensified treatment strategies. However, even if the dual nature of these tumors is fully recognized, there exists no unifying concept of how to treat colorectal mixed adenoneuroendocrine carcinoma patients.

To gain a better understanding of the dual nature of colorectal mixed adenoneuroendocrine carcinomas, it is essential to study their genetic profile. Although much is known about the genetics of conventional colorectal carcinomas ${ }^{10-12}$ and colorectal, as well as pancreatic neuroendocrine neoplasms, ${ }^{13,14}$ data on the most important genetic aberrations of colorectal mixed adenoneuroendocrine carcinomas is still limited. Small case studies on the molecular properties of colorectal and/or gastrointestinal mixed adenoneuroendocrine carcinomas mainly focused on their histogenetic origin $^{15-17}$ and restricted their analyses to only a few genes using conventional sequencing methods. ${ }^{13,15}$ The largest study on these tumors, performed by Sahnane in 2015, concentrated mainly on microsatellite instability. ${ }^{18}$ A comprehensive analysis of the molecular features of colorectal mixed adenoneuroendocrine carcinomas compared with those of colorectal adenocarcinomas and colorectal poorly differentiated neuroendocrine neoplasms, ie, colorectal neuroendocrine carcinomas, is therefore still lacking.

We present a study on the genetic changes in 19 colorectal mixed adenoneuroendocrine carcinomas and eight colorectal neuroendocrine carcinomas, performing targeted next-generation sequencing using a custom-designed colorectal adenocarcinoma-specific panel. In addition, the microsatellite status of colorectal mixed adenoneuroendocrine carcinomas was determined. The questions we addressed focused on (i) differences and similarities in the mutational profiles of colorectal mixed adenoneuroendocrine carcinomas compared with those of conventional colorectal adenocarcinomas on the one hand and to neuroendocrine carcinomas on the other hand, (ii) shared mutations of the exocrine and neuroendocrine neoplastic components, (iii) potential associations of the histopathology of mixed adenoneuroendocrine carcinomas with their mutational profiles, and (iv) the recognition of potential therapeutic molecular targets in colorectal mixed adenoneuroendocrine carcinomas.

\section{Materials and methods}

\section{Sample Recruitment}

We retrospectively analyzed 19 primary colorectal mixed adenoneuroendocrine carcinomas and eight colorectal neuroendocrine carcinomas, retrieved from the Institute of Pathology and the Consultation Center for Pancreatic and Endocrine Tumors, Technical University of Munich, and the Institute of Pathology, Charité University Medicine, Berlin. All the specimens were formalin-fixed, paraffinembedded, and stained with hematoxylin and eosin and Periodic acid Schiff for histological analysis. Colorectal mixed adenoneuroendocrine carcinoma was diagnosed if the respective neoplasms showed coexisting exocrine and neuroendocrine differentiation, with each component representing at least $30 \%$ of the total tumor cell population. In three mixed adenoneuroendocrine carcinomas $(16 \%)$, in which the two tumor components showed a mosaic pattern and could be easily distinguished from each other, respective areas were marked and separately microdissected from unstained slides. All the samples with a tumor cell content of more than $30 \%$ were considered suitable for genetic testing. Colorectal neuroendocrine carcinomas were diagnosed according to World Health Organization criteria and the tumors were subclassified into large cell and small cell types. ${ }^{4}$ This study has been approved by the local ethic committee of the Technical University of Munich (reference number: 252/16 s).

\section{Immunohistochemistry}

Three micrometer-thick paraffin sections were used for immunohistochemical stainings of colorectal mixed adenoneuroendocrine carcinomas on a fully automated slide preparation system ('Benchmark XT System'; Ventana Medical Systems, Tucson, AZ, USA) using reagents and buffers from Ventana Medical Systems. Immunohistochemical stainings were performed using antibodies against synaptophysin (Ventana Medical Systems; 1:1), chromogranin A (Boehringer, Mannheim, Germany; 1:5000), Ki67 (DakoCytomation, Glostrup, Denmark; 1:50), p53 (DakoCytomation; 1:200), Somatostatin receptor 2A (ZYTOMED Systems, Berlin, Germany; 1:100), MUC-1 (Leica Biosystems, Nussloch, Germany; 1:50), MUC-2 (Santa Cruz, Dallas, TX, USA; 1:150), Rb1 (BD Biosciences, Heidelberg, Germany; 1:200), and cytokeratin 20 (PROGEN Biotechnik GmbH, Heidelberg, Germany; 1:60). The stainings were scored as specific in cases of nuclear (Ki-67, p53, Rb1), cytoplasmic (synaptophysin, chromogranin A, MUC-1, MUC-2, cytokeratin 20), and membranous (Somatostatin receptor 2A) positivity. The Ki-67 index was scored by observing a minimum of 500 neoplastic cells in hotspot areas independently by three pathologists (GK, MJ, and BK). Nuclear p53 expression was scored as abnormal in cases of complete loss or in cases of moderate and strong positivity in more than $20 \%$ of neoplastic cells. Rb1 staining was considered as abnormal in cases of nuclear loss in more than $90 \%$ of neoplastic cells. Somatostatin receptor $2 \mathrm{~A}$ expression was evaluated 
using the established human epidermal growth factor receptor2 scoring system of the breast. ${ }^{19}$

\section{DNA Preparation}

Using the Maxwell 16 Research System (Promega, Madison, WI, USA), genomic DNA was extracted after proteinase $\mathrm{K}$ digestion by fully automated purification. Using the QuBit HS DNA Assay (Thermo Fisher Scientific, Waltham, MA, USA), DNA content was measured fluorimetrically. Realtime qPCR-based method (RNAseP Detection system, Thermo Fisher Scientific) confirmed sequencing grade quality. ${ }^{20,21}$

\section{Microsatellite Instability Analysis}

Microsatellite instability typing of colorectal mixed adenoneuroendocrine carcinomas was performed using the marker panel BAT25, BAT26, D5S346, D2S123, and D17S250, as described previously. ${ }^{22}$ High microsatellite instability was scored if at least two of five markers showed genetic instability. MLH-1 methylation status of colorectal mixed adenoneuroendocrine carcinomas was determined using methylation-quantification of endonuclease-resistant DNA as described previously. ${ }^{23}$

\section{Library Preparation and Semiconductor Sequencing}

As described previously, the multiplex PCR-based Ion Torrent AmpliSeq technology (Thermo Fisher Scientific) combined with our custom-designed Colorectal Cancer Panel was used for library preparation. ${ }^{24-26}$ This panel was modified for our study by including 16 amplicons covering hotspot regions from $R B 1$ and $R E T$, respectively, known to be affected in neuroendocrine neoplasms (for details, see Supplementary Table 1). Amplicon library preparation was performed using the Ion AmpliSeq Library Kit v2.0. This panel consists of one primer pool yielding 196 amplicons covering hotspot regions of 32 genes. Approximately $10 \mathrm{ng}$ of DNA, determined by qPCR assay, were used for amplification. Library preparation was performed according to the manufacturers protocol (Thermo Fisher Scientific), as described previously. ${ }^{24-26}$ The individual libraries were diluted to a final concentration of $100 \mathrm{pM}$. Using the Ion PGM Template OT2 200 Kit, all libraries were pooled and processed on Ion Spheres for library amplification. Quality control was performed for unenriched libraries using Ion Sphere quality control measurement on a QuBit 2.0 instrument. Following library enrichment (Ion OneTouch ES, Thermo Fisher Scientific), the Ion PGM Sequencing 200 Kit v2 chemistry was used to process the library for sequencing on a $318 \mathrm{v} 2$ chip loaded with 10-12 barcoded libraries.

\section{Data Analysis}

The implemented Torrent Suite Software (version 4.4.3) was used for processing of raw sequencing data and alignment against the human genome (version hg19) was performed using the Torrent Mapping Alignment Program algorithm. The buildin Variant Caller plugin (version 4.4.3) was used for variant calling. Variant annotation was performed using ANNOVAR. Sequencing reads were visualized using the Integrative Genomics Viewer Browser (IGV, http://www.broadinstitute.org/igv/). To identify already known somatic mutations and mutation types, variants were screened against the Catalogue of Somatic Mutations in Cancer database. ${ }^{27}$ To exclude common germline variants from the analysis, the single-nucleotide polymorphism database ${ }^{28}$ and the Exome Variant Server (http://evs.gs.washing ton.edu/EVS/) were screened.

\section{Prediction of Copy Number Variations}

Detection and identification of copy number variations (amplifications and deletions) was performed using the coverage plugin integrated in the Torrent Suite software analyzing the coverage data summary for each sample and each amplicon, as described previously. ${ }^{21,29}$

\section{CRC Control Group}

The genetic data generated for colorectal mixed adenoneuroendocrine carcinomas and colorectal neuroendocrine carcinomas were compared with a large data set from 196 colorectal adenocarcinomas, which were genetically investigated by a similar colorectal adenocarcinoma-specific panel. ${ }^{26}$

\section{Statistical Analysis}

Statistical analysis was performed using the Statistic Package for Social Sciences 23.0 statistical software (SPSS, Chicago, IL, USA). Correlation analyses between genetic data and clinicopathological parameters were performed using Fisher's exact test. A $P$ value $<0.05$ was considered as significant.

\section{Results}

\section{Clinicopathologic Features of Colorectal Mixed Adenoneuroendocrine Carcinomas and Neuroendocrine Carcinomas}

Our tumor series included 19 colorectal mixed adenoneuroendocrine carcinomas and eight colorectal neuroendocrine carcinomas. As summarized in Table 1, most mixed adenoneuroendocrine carcinomas were locally advanced $(\geq \mathrm{pT} 3=90 \%)$ and showed extensive lymphovascular invasion (L1+/V1 $+=84 \%$ ). There were three mixed adenoneuroendocrine carcinomas (16\%) showing a mosaic 
('collision') pattern, with formations of a large-cell neuroendocrine carcinoma lying side-by-side to an adenocarcinoma component composed of either tubular (1/3) or cribriform (2/3) glandular structures. Fourteen mixed adenoneuroendocrine carcinomas $(74 \%)$ showed a combined pattern with a predominantly cribriform adenocarcinoma and/or mucinous/ signet ring cell pattern intermingled with poorly differentiated neuroendocrine cells. Two mixed adenoneuroendocrine carcinomas with a combined pattern harbored a well-differentiated neuroendocrine component and were provisionally termed as mixed adenoneuroendocrine tumors (Figure 1). A small-cell neuroendocrine carcinoma component was not identifiable in any of the mixed adenoneuroendocrine carcinoma cases.

All colorectal neuroendocrine carcinomas were only composed of poorly differentiated neuroendocrine cells. Six out of eight neuroendocrine carcinomas $(75 \%)$ were localized in the colon and two had a rectal localization. All neuroendocrine carcinomas were of large-cell type $(7 / 8 ; 88 \%)$, except for one, which showed a small-cell pattern and resided in the colon.

\section{Immunohistochemical Analysis of Colorectal Mixed Adenoneuroendocrine Carcinomas}

All tumors stained for synaptophysin in at least 30\% of the tumor cells. Chromogranin A was positive in $13 / 19$ tumors. The proliferative activity of the neuroendocrine tumor components labeled by nuclear Ki-67 expression, varied from $30 \%$ to $90 \%$ in mixed adenoneuroendocrine carcinomas, while the two mixed adenoneuroendocrine tumors harbored a neoplastic endocrine component with a proliferation index of $<20 \%$ (G2). Abnormal immunohistochemical p53 expression was present in 10/19 tumors (53\%) and showed a strong correlation with concurrent somatic TP53 mutations $(P=0.001)$. Nuclear Rb1 expression was completely lost in two tumors and retained, sometimes patchy, in the remaining 16 tumors (88\%). All mixed adenoneuroendocrine carcinomas stained for cytokeratin 20, while MUC-1 and MUC-2 were each focally expressed in $12 / 19$ mixed adenoneuroendocrine carcinomas. Membranous somatostatin receptor $2 \mathrm{~A}$ staining (score $2+/ 3+$ ) was not detected in any of the tumors.

\section{Genetic Profile of Colorectal Mixed Adenoneuroendocrine Carcinomas}

Our targeted next-generation sequencing approach identified 43 somatic mutations in 13 of the 32 colorectal adenocarcinoma and poorly differentiated neuroendocrine neoplasm-related genes included in our customized colorectal adenocarcinoma-specific panel (mean coverage: 2530 (range: 205-9546), mean total reads: 483441 (range: 172 841-1 369651 )). We
Table 1 Clinicopathological features of 19 colorectal mixed adenoneuroendocrine carcinomas

\begin{tabular}{lc}
\hline Clinicopathological features & No. $(\%)$ \\
\hline Patients & 19 \\
Male & $11(58 \%)$ \\
Female & $8(42 \%)$ \\
Age at diagnosis & \\
Mean & 64.3 \\
Range & $34-96$ \\
Tumor localization & \\
Colon & $17(90 \%)$ \\
Rectum & $2(10 \%)$ \\
pT-stage & \\
I-II & $2(10 \%)$ \\
III & $14(74 \%)$ \\
IV & $3(16 \%)$ \\
Lymphovascular invasion & $16(84 \%)$ \\
Tumor histopathology & \\
I Mosaic ('collision') type & $3(16 \%)$ \\
II Composite type & $14(74 \%)$ \\
III Mixed adenoneuroendocrine tumor & $2(10 \%)$ \\
Grading of exocrine component & \\
G1 & \\
G2 & \\
G3 & \\
Grading of endocrine component & $17(37 \%)$ \\
G1 & \\
G2 & \\
G3 & \\
\hline & \\
& \\
&
\end{tabular}

detected genetic alterations in 16 of 19 (84\%) investigated colorectal mixed adenoneuroendocrine carcinomas with allele frequencies varying from 5 to $73 \%$ (Figure 2). Sixteen percent (7/43) of the identified variants in our mixed adenoneuroendocrine carcinoma cohort have not yet been reported to the Catalogue of Somatic Mutations in Cancer database at the time of data query. Microsatellite analysis revealed high microsatellite instability in two mixed adenoneuroendocrine carcinomas (11\%), which was associated with $M L H-1$ promoter hypermethylation in both cases displaying high microsatellite instability (100\%) (Figure 2). Singular copy number variations were present in two genes (RB1, $M Y C$ ) in two different cases.

Genes Commonly Associated with Colorectal Adenocarcinoma Carcinogenesis. Somatic mutations of the TP53 gene were most frequent ( $9 / 19$ cases; $47 \%$ ) and affected all exons. TP53 mutations were not detected in tumors displaying high microsatellite instability, but this finding was not statistically significant $(P=0.283)$. BRAF mutations $(7 / 19$ cases; $37 \%$ ) were the second most common alteration and exclusively located in Exon 15, primarily leading to p.V600E (86\%). Most BRAF mutant mixed 

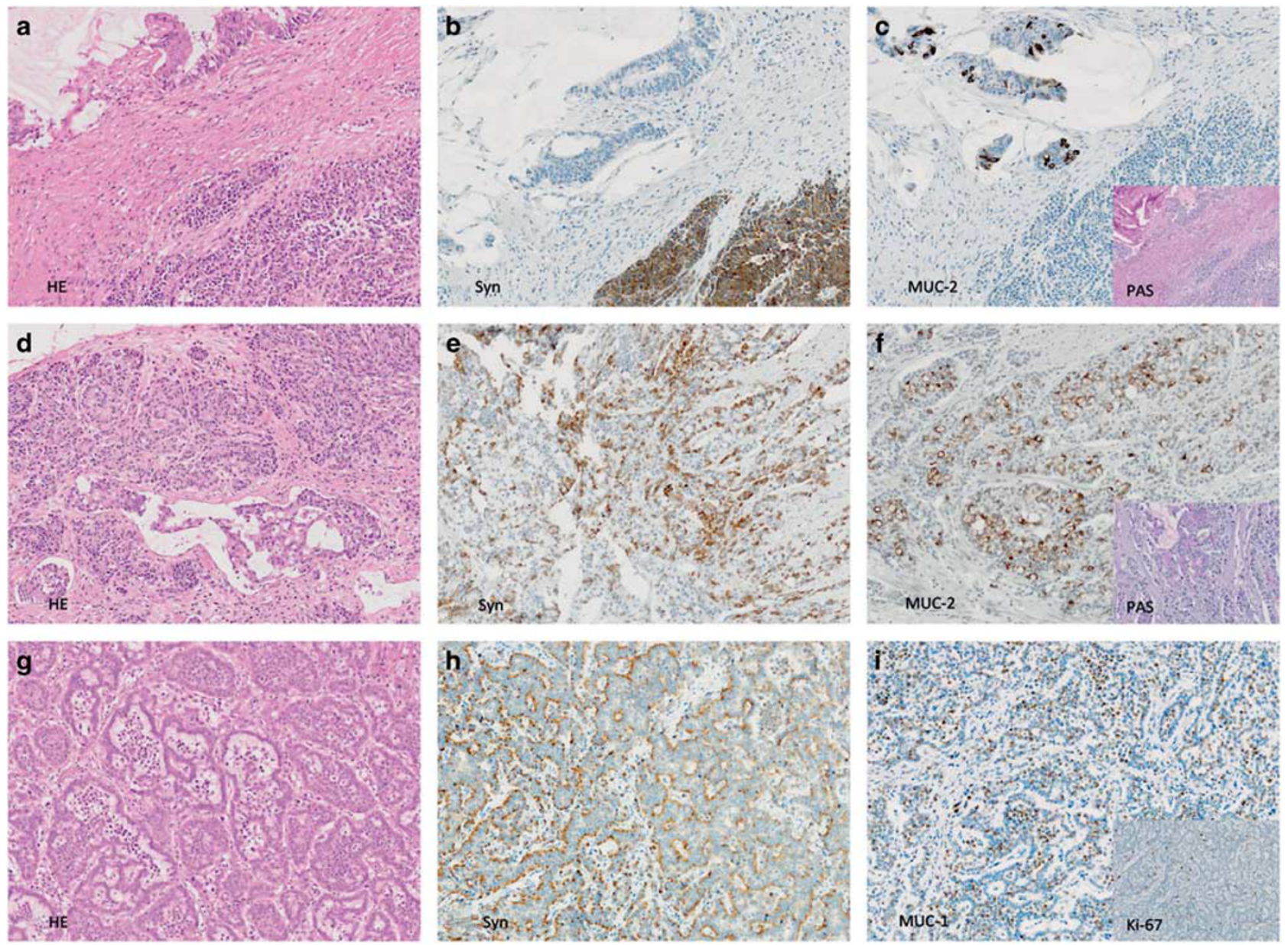

Figure 1 Histologic growth patterns in colorectal mixed adenoneuroendocrine carcinomas. (a-c) Mosaic ('collision') pattern (a) with expression of synaptophysin (b), MUC-2 (c), and Periodic acid Schiff positivity (c, inset). (d-f) Combined (composite) pattern (d) with expression of synaptophysin (e), MUC-2 (f), and Periodic acid Schiff positivity (f, inset). (g-i) Colorectal adenocarcinoma combined with a neuroendocrine tumor G2 (g) with expression of synaptophysin (h), MUC-1 (i), and a Ki-67 index $<20 \%$ (i, inset).

adenoneuroendocrine carcinomas were KRAS wildtype $(85 \%)$ and showed a positive association with coexisting ATM mutations $(3 / 19$ cases; $14 \%$; $P=0.023)$, but not with high microsatellite instability $(P=0.123)$ or a signet ring/mucinous tumor phenotype $(P=0.5)$. Four of the 19 mixed adenoneuroendocrine carcinomas $(21 \%)$ harbored KRAS mutations which were distributed over three exons (Ex 2: 50\%; Ex 3: 25\%; Ex 4: 25\%). One microsatellite stable tumor harbored two concurrent KRAS mutations (case \#14: p.G12S; allele frequency: 28\%; p.A146T; allele frequency: $11 \%$ ) as well as a coexisting, non-p. V600E BRAF variant (p.D594G; allele frequency: $27 \%$ ). Forty percent of the KRAS wild-type mixed adenoneuroendocrine carcinomas (6/15) displayed a detectable variant in genes (BRAF and PTEN) linked to attenuated therapeutic effect or therapy resistance of anti-epidermal growth factor receptor therapy. $A P C$ was mutated in only three mixed adenoneuroendocrine carcinomas $(16 \%)$, with one tumor harboring two distinct $A P C$ mutations (case \#19: p. R876*; p.R1450*). FBXW7 was altered in three cases
(16\%). Mutations in SOX9 were frequent (11\%) but showed no associations with concurrent mutations or clinicopathological features. PTEN alterations were present in $11 \%$ of investigated mixed adenoneuroendocrine carcinomas (2/19), while ERBB2, PIK3CA, SMAD4, TGFBR2, and MSH3 were each mutated singularly (1/19 each; $5 \%$ ). A copy number variation was only found in one tumor (case \#19), who showed a MYC amplification. Three mixed adenoneuroendocrine carcinomas, two composed of a cribriform adenocarcinoma combined with a largecell neuroendocrine carcinoma, and the other one composed of an adenocarcinoma combined with a well-differentiated neuroendocrine neoplasm component ('mixed adenoneuroendocrine tumor'), lacked any mutations.

Genes Commonly Associated with Poorly Differentiated Neuroendocrine Neoplasms. Mixed adenoneuroendocrine carcinomas showed no somatic $R B 1$ or RET mutations, but a heterozygous $R B 1$ deletion was revealed by copy number variation analysis in 


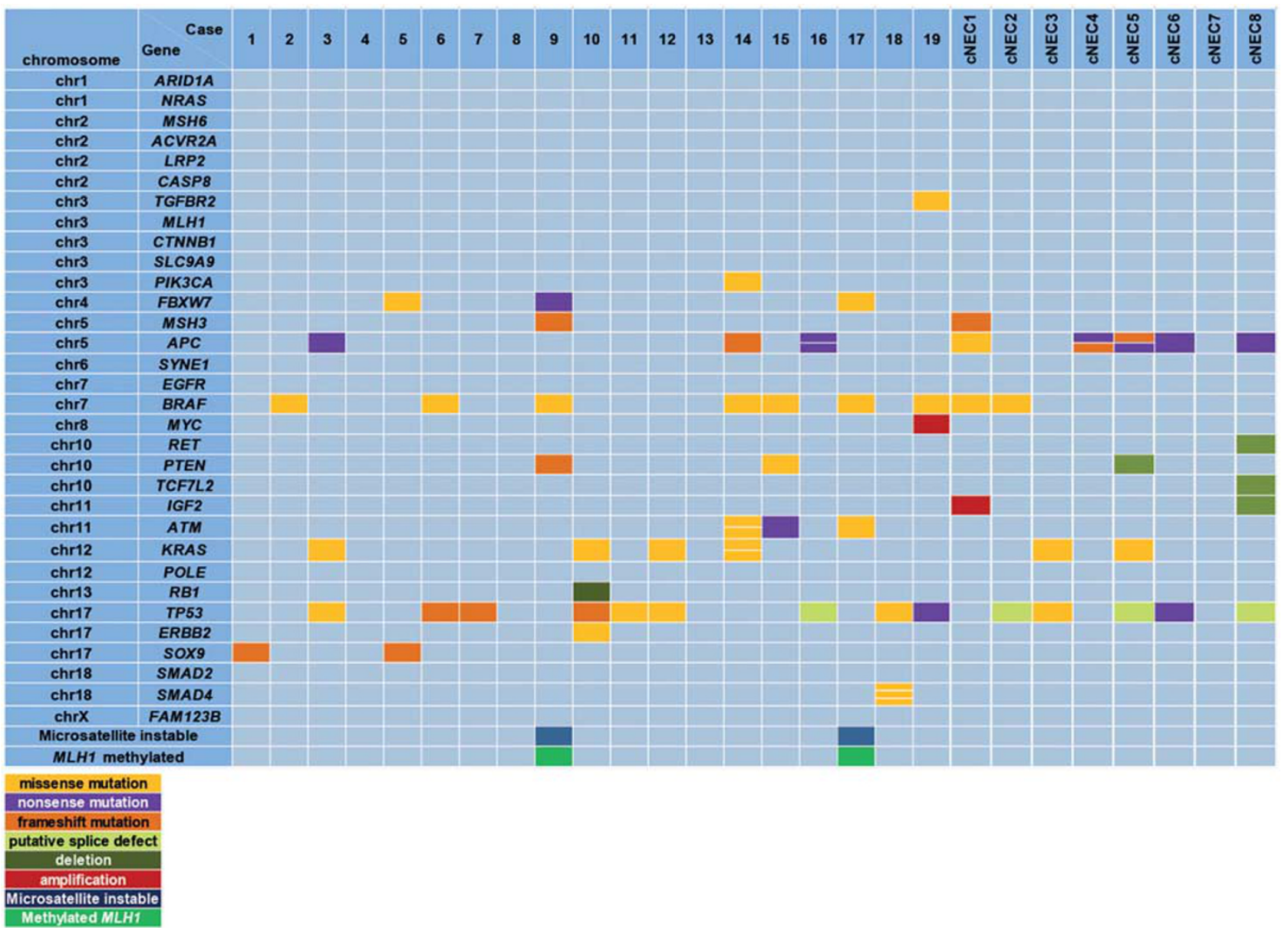

Figure 2 Detailed genotyping data of 19 mixed adenoneuroendocrine carcinomas and eight colorectal neuroendocrine carcinomas showing non-synonymous mutations and microsatellite status in 32 genes related to colorectal adenocarcinoma. Each column of the matrix represents one mixed adenoneuroendocrine carcinoma or colorectal neuroendocrine carcinoma. Each colored box represents variants identified in the samples. A split of a box indicates that more than one variant was identified in the same gene (one split=two variants; two splits = three variants). Color annotations: dark yellow: missense mutation; pink: nonsense mutation; dark orange: frameshift; light green: splice defect; dark green: deletion; red: amplification; dark blue: high microsatellite instability; green: methylated $M L H-1$.

one case (\#10). RET copy number variations were not found.

Separate Genetic Profiling of Exo- and Neuroendocrine Components. In three tumors, it was possible to analyze the exocrine and neuroendocrine carcinoma components separately (cases \#15, \#16, \#18). As illustrated in Figure 3, all separately investigated tumor components shared a mutational trunk of somatic variants with high allele frequencies in wellcharacterized driver genes related to colorectal adenocarcinoma carcinogenesis (eg, TP53, BRAF), with only slight allele frequency-deviations between the morphologically different neoplastic components. Case \#15 presented a low-frequent ATM mutation (p.R2443*; allele frequency: $4 \%$ ), which was confined to the exocrine component, while a supplementary low-frequent SMAD4 variant (p.W524L; allele frequency: 8\%) was detectable in the exocrine component of case \#18.

\section{Genetic Profile of Colorectal Neuroendocrine Carcinomas}

Seven of the eight neuroendocrine carcinomas (88\%) harbored somatic mutations. In total, 17 somatic mutations were identified, which were detected in 5 of the 32 genes implemented in our panel with allele frequencies varying from 31 to $89 \%$. APC and TP53 gene alterations were most frequent ( $5 / 8$ neuroendocrine carcinomas each, $63 \%$; including the small cell neuroendocrine carcinoma), with two neoplasms harboring two coexisting APC mutations. BRAF (p.V600E) and KRAS variants were present in two cases, respectively (25\%), while an MSH3 mutation was detected in one neuroendocrine carcinoma (13\%). Copy number variation analysis revealed a total of four deletions affecting RET, PTEN, TCF7L2, and IGF2, which were identified in two neoplasms. One neuroendocrine carcinoma showed an IGF2 amplification. One large-cell neuroendocrine carcinoma had no detectable genomic alterations (Figure 2). 


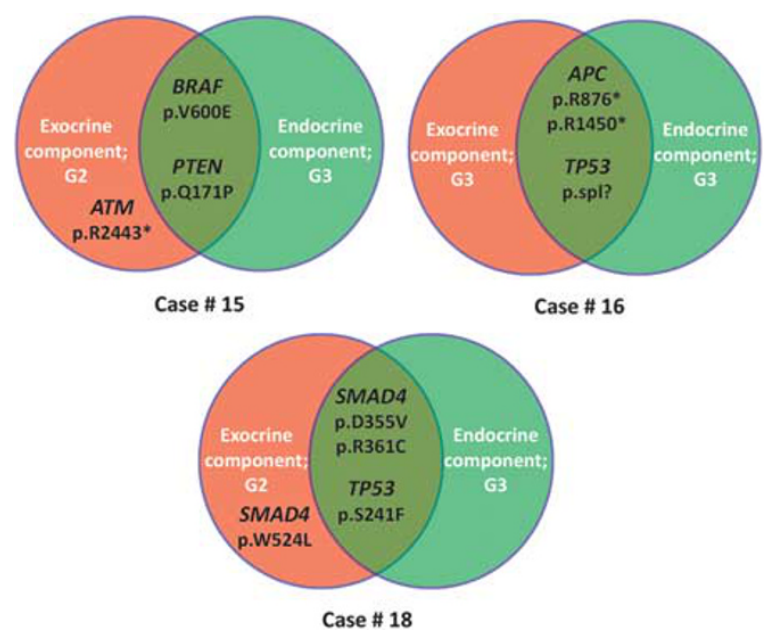

Figure 3 Schematic illustration of the shared mutational trunk in three cases of colorectal mixed adenoneuroendocrine carcinoma. Paired circles show either the mutations in the exocrine (red color; exocrine) or the neuroendocrine (green color; endocrine) component. The overlapping areas highlight the shared mutations.

\section{Comparison of the Mutation Profiles of Colorectal Mixed Adenoneuroendocrine Carcinomas and Neuroendocrine Carcinomas with that of Colorectal Adenocarcinomas}

Compared with the data generated for colorectal adenocarcinomas, ${ }^{26}$ the rate of $A P C(P=0.001)$ and KRAS $(P=0.043)$ mutations was significantly lower than in colorectal mixed adenoneuroendocrine carcinomas, while $B R A F$ variants $(P=0.006)$ were more frequent. Compared with neuroendocrine carcinomas, mixed adenoneuroendocrine carcinomas had less APC mutations than neuroendocrine carcinomas $(P=0.027)$, while there were no significant differences in the mutation rates between neuroendocrine carcinomas and colorectal adenocarcinomas (Figure 4).

\section{Discussion}

In this study, we examined 19 colorectal mixed adenoneuroendocrine carcinomas and eight neuroendocrine carcinomas by targeted next-generation sequencing and compared the results with data from a large colorectal adenocarcinoma cohort of 196 patients, which we recently obtained by using a similar colorectal adenocarcinoma-specific panel. ${ }^{26}$ Our analysis revealed that both, mixed adenoneuroendocrine carcinomas and neuroendocrine carcinomas, have a mutation profile that is similar to that of colorectal adenocarcinomas, indicating a shared genetic framework of these rare tumor entities with conventional colorectal adenocarcinomas, a view that is also supported by data from earlier studies. ${ }^{13,15,30,31}$

As illustrated in Figure 4, colorectal mixed adenoneuroendocrine carcinomas showed frequent mutations in genes classically linked to colorectal adenocarcinoma carcinogenesis. Interestingly, however, the frequencies of the $A P C, K R A S$, and $B R A F$ mutations differed significantly from those in conventional colorectal adenocarcinomas. Although $A P C \quad(P=0.001)$ and KRAS $(P=0.043)$ mutations showed lower frequencies, a distinctly higher rate was found for $B R A F$ alterations $(P=0.006) .^{10,26}$ $B R A F$ mutations may therefore play a special role in the development of colorectal mixed adenoneuroendocrine carcinomas and also seem to be associated with poor outcome, as it has been reported recently. ${ }^{30,31}$

Compared with poorly differentiated neuroendocrine neoplasms, also called neuroendocrine carcinomas, it is striking that mixed adenoneuroendocrine carcinomas virtually lacked alterations in genes commonly related to neuroendocrine carcinomas from various organ sites, such as RB1 and RET. ${ }^{32-35}$ Only one microsatellite stable tumor harbored an isolated $R B 1$ deletion. Furthermore, only $49 \%$ of our mixed adenoneuroendocrine carcinoma cohort harbored TP53 mutations, a frequency far lower than previously described for poorly differentiated neuroendocrine neoplasms of the pancreas. ${ }^{14}$

Surprisingly, when we analyzed the genetic profile of our colorectal neuroendocrine carcinomas, we found high mutation rates of the colorectal adenocarcinoma-associated genes $A P C, K R A S$, $B R A F$, and TP53. APC mutations were even more frequent in colorectal neuroendocrine carcinomas than in colorectal mixed adenoneuroendocrine carcinomas $(P=0.027)$. It seems therefore that not only colorectal mixed adenoneuroendocrine carcinomas, but also colorectal neuroendocrine carcinomas have a developmental relationship to conventional colorectal adenocarcinomas, a notion, that has also been put forward in two other recent studies..$^{30,31}$

Among our series of mixed adenoneuroendocrine carcinomas, there are two tumors that showed a well-differentiated neuroendocrine cell component instead of poorly differentiated neuroendocrine cells, and were therefore provisionally classified as mixed adenoneuroendocrine tumors. Although one of the two mixed adenoneuroendocrine tumors had no mutations, rendering any statement regarding a relationship to colorectal adenocarcinomas or neuroendocrine neoplasms impossible, the other mixed adenoneuroendocrine tumor, like many of the mixed adenoneuroendocrine carcinomas, showed mutations in driver genes linked to colorectal adenocarcinoma carcinogenesis, and displayed no genetic connection to neuroendocrine neoplasms. The last finding, however, has to be interpreted with caution, as specific genetic alterations of well-differentiated neuroendocrine neoplasms of the colorectum are not known so far and genes such as ATRX, DAXX, or $M E N 1$, which were frequently found to be mutated in well-differentiated neuroendocrine neoplasms of the pancreas, ${ }^{36}$ were not included in our panel.

The fact that many colorectal mixed adenoneuroendocrine carcinomas and most neuroendocrine carcinomas are genetically intimately connected to 


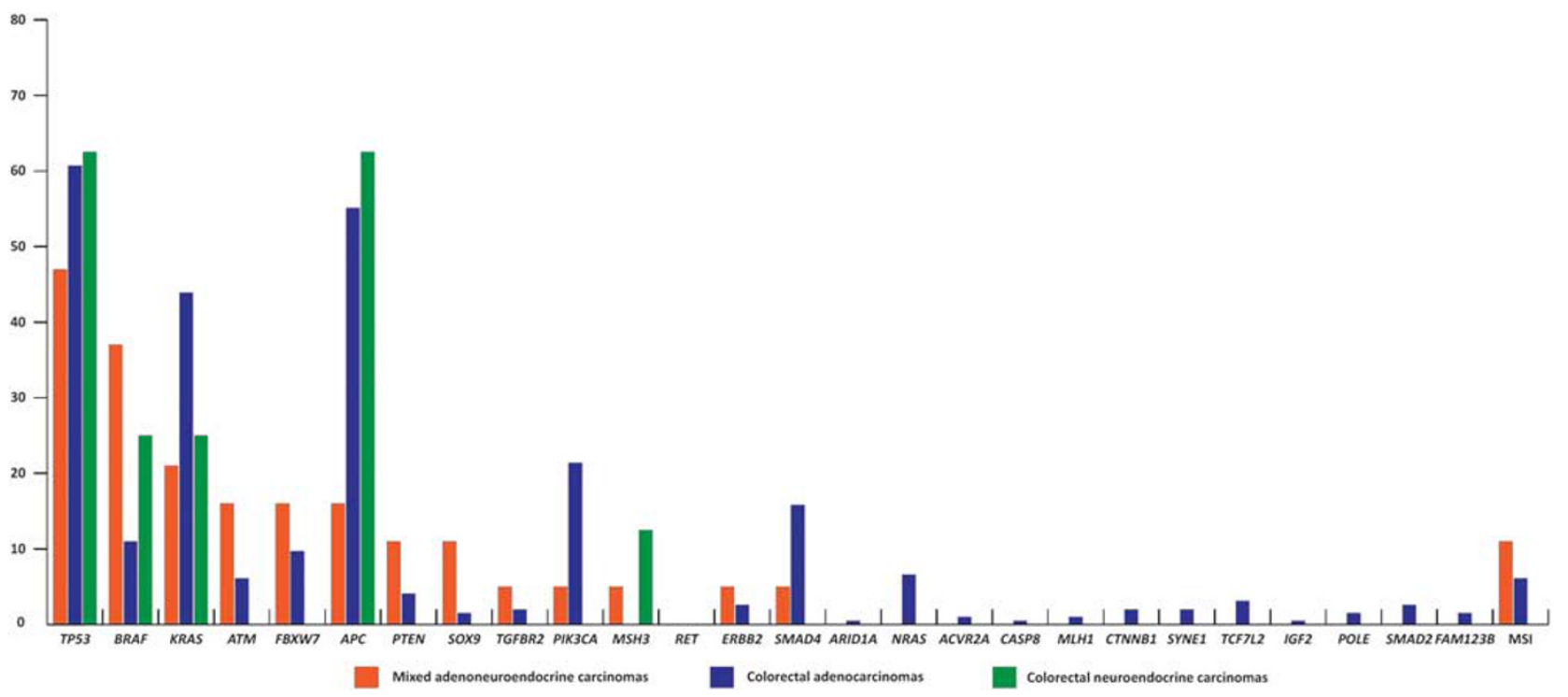

Figure 4 Relative frequencies of mutations in colorectal mixed adenoneuroendocrine carcinomas and colorectal neuroendocrine carcinomas compared with each other and with those of a large cohort of colorectal adenocarcinomas. ${ }^{26}$ Note the frequent somatic mutations in colorectal adenocarcinoma driver genes and BRAF gene variants, and the low frequency of KRAS and APC mutations.

colorectal adenocarcinomas leads to the question how these tumors acquire their neuroendocrine features. On the basis of the data obtained from small series of diverse gastrointestinal mixed adenoneuroendocrine carcinomas, using small gene panels, the hypothesis of a common ancestry with conventional adenocarcinomas has been advanced. ${ }^{13,15,17}$ Our data regarding the three mixed adenoneuroendocrine carcinomas, which exhibited a mosaic pattern and whose two components could therefore be separately analyzed also suggest that the exocrine and neuroendocrine neoplastic components in colorectal mixed adenoneuroendocrine carcinomas share a mutational trunk (see Figure 3). It may therefore be speculated that colorectal mixed adenoneuroendocrine carcinomas and neuroendocrine carcinomas may arise from a common multipotent intestinal stem cell clone. Later during their development, the neoplasms neuroendocrine differentiation may either arise secondarily owing to epigenetic, transcriptional, or translational events, or may be based on cumulatively acquired genetic alterations in genes not included in our panel.

The mutational profile in colorectal mixed adenoneuroendocrine carcinomas is not homogeneous. Only up to $30 \%$ of the tumors harbor mutations in two or three of the four genes APC, BRAF, KRAS, and TP53 and up to $15 \%$ of them show mutations in genes such as ATM, FBXW7, or SOX9, which are relatively infrequent in colorectal adenocarcinomas and absent in neuroendocrine carcinomas. As these genetic findings may be due to tumor heterogeneity, and histological heterogeneity is a striking finding in colorectal mixed adenoneuroendocrine carcinomas, ${ }^{3,5}$ we tried to find a genotype-phenotype correlation. Roughly, three histologic subtypes may be distinguished. First, mixed adenoneuroendocrine carcinomas with a mosaic ('collision') pattern in which the two components, a glandular adenocarcinoma and a neuroendocrine carcinoma, form complexes that are usually separated by small connective tissue bands. Second, mixed adenoneuroendocrine carcinomas with a combined ('composite') pattern in which a cribriform (rarely a mucinous or signet ring cell type) adenocarcinoma intermingles with a neuroendocrine carcinoma, and third, mixed adenoneuroendocrine tumors in which an adenocarcinoma combines with a well-differentiated neuroendocrine neoplasm component. ${ }^{6,37}$ The first and the latter subtype were only recognized in three and two tumors, respectively. When we correlated the three subtypes with their respective molecular changes, we found no distinct genotype-phenotype pattern. In colorectal adenocarcinoma, for instance, it is known that mucinous/signet ring cell carcinomas show high frequencies of $B R A F$ mutations and microsatellite instability. ${ }^{26,38}$ Those features, however, were not present in the respective mixed adenoneuroendocrine carcinomas of our cohort. Furthermore, no distinct differences in the mutational profile of mixed high-grade vs low-grade mixed adenoneuroendocrine carcinomas, ie, mixed adenoneuroendocrine tumors were detectable. These data, however, have to be interpreted with caution because the correlations are based on a rather low number of tumors and highly variable mutation patterns.

If colorectal mixed adenoneuroendocrine carcinomas (and neuroendocrine carcinomas) are closely related to colorectal adenocarcinomas, it is of interest whether there are molecular alterations in these tumors that indicate a potential response to colorectal adenocarcinoma-directed drugs. The comparatively low frequency of KRAS mutations in colorectal mixed adenoneuroendocrine carcinomas and neuroendocrine carcinomas indicates a potential 
efficacy of anti-epidermal growth factor receptor therapy in these neoplasms. This view is supported by a recent case report of a patient with a KRAS wildtype mixed adenoneuroendocrine carcinoma of the rectum, who was successfully treated with Cetuximab. ${ }^{39}$ Moreover, the high rate of BRAF alterations in colorectal mixed adenoneuroendocrine carcinomas, with or without concurrent high microsatellite instability, is also important. Some colorectal adenocarcinomas harboring these alterations seem to have a poor patient outcome. ${ }^{40-42}$ However, they are thought to be a promising target of combined rapidly accelerated fibrosarcoma inhibitor and antiepidermal growth factor receptor therapy, ${ }^{43}$ but not of a singular anti-epidermal growth factor receptor therapy. ${ }^{44}$ Microsatellite instability, a wellcharacterized feature of colorectal adenocarcinoma with prognostic ${ }^{45}$ and therapeutic relevance, was diagnosed in a similar proportion in colorectal mixed adenoneuroendocrine carcinomas compared with conventional colorectal adenocarcinomas, and was present in exocrine and endocrine tumor components, raising the question of possible immune checkpoint inhibitor therapy in this distinct subpopulation of mixed colorectal neoplasms displaying high microsatellite instability. ${ }^{46}$

In summary, our study reveals a genetic affiliation of most of the colorectal mixed adenoneuroendocrine carcinomas and colorectal neuroendocrine carcinomas to the colorectal adenocarcinoma family, thereby supporting the hypothesis of a common ancestry of both exocrine and neuroendocrine neoplastic components in many of these tumors. The demonstrated colorectal adenocarcinoma-like mutational profile in colorectal mixed adenoneuroendocrine carcinomas additionally highlights certain drugable targets, pointing towards a potential effectiveness of molecular based treatment options, eg, anti-epidermal growth factor receptor therapy, in this rare tumor entity.

\section{Acknowledgments}

We thank Kathrin Ridinger, Petra Meyer, and Sandra Hiltensberger for excellent technical assistance. We thank Philipp Lohneis who has supported this study. This study has been supported by a fund of the German Cancer Consortium (to WW).

\section{Disclosure/conflict of interest}

The authors declare no conflict of interest.

\section{References}

1 Volante M, Rindi G, Papotti M. The grey zone between pure (neuro)endocrine and non-(neuro)endocrine tumours: a comment on concepts and classification of mixed exocrine-endocrine neoplasms. Virchows Arch 2006;449:499-506.

2 La Rosa S, Marando A, Furlan D, Sahnane N, Capella C. Colorectal poorly differentiated neuroendocrine carcinomas and mixed adenoneuroendocrine carcinomas: insights into the diagnostic immunophenotype, assessment of methylation profile, and search for prognostic markers. Am J Surg Pathol 2012;36:601-611.

3 La Rosa S, Marando A, Sessa F, Capella C. Mixed adenoneuroendocrine carcinomas (MANECs) of the gastrointestinal tract: an update. Cancers 2012;4:11-30.

4 Rindi G, Arnold R, Bosman FT, et al. Nomenclature and classification of neuroendocrine neoplasms of the digestive system. In: Bosman FT, Carneiro F, Hruban $\mathrm{RH}$, Theise ND (eds). WHO Classification of Tumours of the Digestive System, 4th edn. WHO Press, IARC: Lyon, France, 2010, pp 13-14.

5 Kloppel G. [Neoplasms of the disseminated neuroendocrine cell system of the gastrointestinal tract]. Der Pathologe 2015;36:237-245.

6 Li Y, Yau A, Schaeffer D, et al. Colorectal glandularneuroendocrine mixed tumor: pathologic spectrum and clinical implications. Am J of Surg Pathol 2011;35:413-425.

7 Komatsubara T, Koinuma K, Miyakura Y, et al. Endocrine cell carcinomas of the colon and rectum: a clinicopathological evaluation. Clin J Gastroenterol 2016;9:1-6.

8 Chen MH, Kuo YJ, Yeh YC, et al. High neuroendocrine component is a factor for poor prognosis in gastrointestinal high-grade malignant mixed adenoneuroendocrine neoplasms. J Chin Med Assoc 2015;78:454-459.

9 Brathwaite S, Rock J, Yearsley MM, et al. Mixed adenoneuroendocrine carcinoma: an aggressive clinical entity. Ann Surg Oncol 2016;23:2281-2286.

10 Cancer Genome Atlas Network. Comprehensive molecular characterization of human colon and rectal cancer. Nature 2012;487:330-337.

11 Fearon ER, Vogelstein B. A genetic model for colorectal tumorigenesis. Cell 1990;61:759-767.

12 Fearon ER. Molecular genetics of colorectal cancer. Annu Rev Pathol 2011;6:479-507.

13 Takizawa N, Ohishi Y, Hirahashi M, et al. Molecular characteristics of colorectal neuroendocrine carcinoma; similarities with adenocarcinoma rather than neuroendocrine tumor. Hum Pathol 2015;46:1890-1900.

14 Yachida S, Vakiani E, White CM, et al. Small cell and large cell neuroendocrine carcinomas of the pancreas are genetically similar and distinct from welldifferentiated pancreatic neuroendocrine tumors. Am J Surg Pathol 2012;36:173-184.

15 Karkouche R, Bachet JB, Sandrini J, et al. Colorectal neuroendocrine carcinomas and adenocarcinomas share oncogenic pathways. A clinico-pathologic study of 12 cases. J Gastroenterol 2012;24:1430-1437.

16 Vanacker L, Smeets D, Hoorens A, et al. Mixed adenoneuroendocrine carcinoma of the colon: molecular pathogenesis and treatment. Anticancer Res 2014;34:5517-5521.

17 Scardoni M, Vittoria E, Volante M, et al. Mixed adenoneuroendocrine carcinomas of the gastrointestinal tract: targeted next-generation sequencing suggests a monoclonal origin of the two components. Neuroendocrinology 2014;100:310-316.

18 Sahnane N, Furlan D, Monti M, et al. Microsatellite unstable gastrointestinal neuroendocrine carcinomas: a new clinicopathologic entity. Endocr Relat Cancer 2015;22:35-45.

19 Wolff AC, Hammond ME, Hicks DG, et al. Recommendations for human epidermal growth factor receptor 2 testing 
in breast cancer: American Society of Clinical Oncology/ College of American Pathologists clinical practice guideline update. J Clin Oncol 2013;31:3997-4013.

20 Stenzinger A, Pfarr N, Penzel R, et al. Semiconductorbased sequencing of formalin-fixed, paraffin-embedded colorectal cancer samples. Oncologist 2015;20:e10-e11.

21 Endris V, Penzel R, Warth A, et al. Molecular diagnostic profiling of lung cancer specimens with a semiconductorbased massive parallel sequencing approach: feasibility, costs, and performance compared with conventional sequencing. J Mol Diagn 2013;15:765-775.

22 Boland CR, Thibodeau SN, Hamilton SR, et al. A National Cancer Institute Workshop on Microsatellite Instability for cancer detection and familial predisposition: development of international criteria for the determination of microsatellite instability in colorectal cancer. Cancer Res 1998;58:5248-5257.

23 Bettstetter M, Dechant S, Ruemmele P, et al. MethyQESD, a robust and fast method for quantitative methylation analyses in HNPCC diagnostics using formalin-fixed and paraffin-embedded tissue samples. Lab Invest 2008;88:1367-1375.

24 Jesinghaus $\mathrm{M}$, Wolf $\mathrm{T}$, Pfarr $\mathrm{N}$, et al. Distinctive spatiotemporal stability of somatic mutations in metastasized microsatellite-stable colorectal cancer. Am J Surg Pathol 2015;39:1140-1147.

25 Jesinghaus M, Pfarr N, Kloor M, et al. Genetic heterogeneity in synchronous colorectal cancers impacts genotyping approaches and therapeutic strategies. Genes Chromosomes Cancer 2016;55:268-277.

26 Jesinghaus M, Pfarr N, Endris V, et al. Genotyping of colorectal cancer for cancer precision medicine: results from the IPH Center for Molecular Pathology. Genes Chromosomes Cancer 2016;55:505-521.

27 Forbes SA, Beare D, Gunasekaran P, et al. COSMIC: exploring the world's knowledge of somatic mutations in human cancer. Nucleic Acids Res 2015;43:D805-D811.

28 Sherry ST, Ward MH, Kholodov M, et al. dbSNP: the NCBI database of genetic variation. Nucleic Acids Res 2001;29:308-311.

29 Pfarr N, Penzel R, Klauschen F, et al. Copy number changes of clinically actionable genes in melanoma, non-small cell lung cancer and colorectal cancer-A survey across 822 routine diagnostic cases. Genes Chromosomes Cancer 2016;55:821-833.

30 Olevian DC, Nikiforova MN, Chiosea S, et al. Colorectal poorly differentiated neuroendocrine carcinomas frequently exhibit BRAF mutations and are associated with poor overall survival. Hum Pathol 2016;49:124-134.

31 Woischke C, Schaaf CW, Yang HM, et al. In-depth mutational analyses of colorectal neuroendocrine carcinomas with adenoma or adenocarcinoma components. Mod Pathol 2016; doi: 10.1038/modpathol.2016. 150 [e-pub ahead of print].

32 Simbolo M, Mian C, Barollo S, et al. High-throughput mutation profiling improves diagnostic stratification of sporadic medullary thyroid carcinomas. Virchows Arch 2014;465:73-78.

33 Rekhtman N, Pietanza MC, Hellmann MD, et al. Nextgeneration sequencing of pulmonary large cell neuroendocrine carcinoma reveals small cell carcinomalike and non-small cell carcinoma-like subsets. Clin Cancer Res 2016;22:3618-3629.

34 Grubbs EG, Ng PK, Bui J, et al. RET fusion as a novel driver of medullary thyroid carcinoma. J Clin Endocrinol Metab 2015;100:788-793.

35 Hedayati M, Zarif Yeganeh M, Sheikholeslami S, et al. Diversity of mutations in the RET proto-oncogene and its oncogenic mechanism in medullary thyroid cancer. Crit Rev Clin Lab Sci 2016;1-11.

36 Jiao Y, Shi C, Edil BH, et al. DAXX/ATRX, MEN1, and mTOR pathway genes are frequently altered in pancreatic neuroendocrine tumors. Science 2011;331: 1199-1203.

37 Lewin K. Carcinoid tumors and the mixed (composite) glandular-endocrine cell carcinomas. Am J Surg Pathol 1987;11:71-86.

38 Chen D, Huang JF, Liu K, et al. BRAFV600E mutation and its association with clinicopathological features of colorectal cancer: a systematic review and metaanalysis. PLoS One 2014;9:e90607.

39 Tani K, Seyama Y, Inada K, et al. [Mixed adenoneuroendocrine carcinoma with multiple liver metastases successfully treated by cetuximab/CPT-11 chemotherapy followed by curative resection - a case report]. Gan To Kagaku Ryoho 2014;41:2145-2147.

40 Yaeger R, Cercek A, Chou JF, et al. BRAF mutation predicts for poor outcomes after metastasectomy in patients with metastatic colorectal cancer. Cancer 2014;120:2316-2324.

41 Ahn TS, Jeong D, Son MW, et al. The BRAF mutation is associated with the prognosis in colorectal cancer. J Cancer Res Clin Oncol 2014;140:1863-1871.

42 Kadowaki S, Kakuta M, Takahashi S, et al. Prognostic value of KRAS and BRAF mutations in curatively resected colorectal cancer. World J Gastroenterol 2015;21:1275-1283.

43 Yaeger R, Cercek A, O'Reilly EM, et al. Pilot trial of combined BRAF and EGFR inhibition in BRAF-mutant metastatic colorectal cancer patients. Clin Cancer Res 2015;21:1313-1320.

44 De Roock W, Claes B, Bernasconi D, et al. Effects of KRAS, BRAF, NRAS, and PIK3CA mutations on the efficacy of cetuximab plus chemotherapy in chemotherapy-refractory metastatic colorectal cancer: a retrospective consortium analysis. Lancet Oncol 2010;11:753-762.

45 Boland CR, Goel A. Microsatellite instability in colorectal cancer. Gastroenterology 2010;138:2073-2087.

46 Le DT, Uram JN, Wang $\mathrm{H}$, et al. PD-1 blockade in tumors with mismatch-repair deficiency. New Engl J Med 2015;372:2509-2520.

Supplementary Information accompanies the paper on Modern Pathology website (http://www.nature.com/ modpathol) 